\title{
MUlTimodal OPTIMAL TRAJECTORY Planning of a Prosthetic Leg
}

\author{
Zlata Jelačić \& Remzo Dedić
}
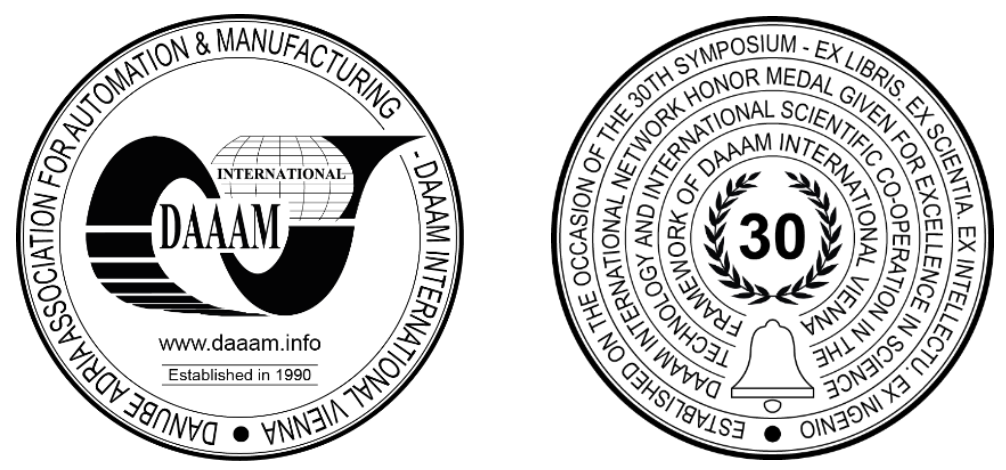

This Publication has to be referred as: Jelacic, Z[lata] \& Dedic, R[emzo] (2019). Multimodal Optimal Trajectory Planning of a Prosthetic Leg, Proceedings of the 30th DAAAM International Symposium, pp.0878-0885, B. Katalinic (Ed.), Published by DAAAM International, ISBN 978-3-902734-22-8, ISSN 1726-9679, Vienna, Austria DOI: $10.2507 / 30$ th.daaam.proceedings. 122

\begin{abstract}
Making even the simplest of moves involves processing a huge amount of information. When we move our extremities to a desired location, there are infinitely many possible trajectories and velocity profiles that a multi-hinged leg could take, and in addition, each trajectory can be achieved from multiple combinations of joint angles. Further, since we have many more muscles than joints, any leg configuration can be generated from infinitely many variations of muscle activation. Biomechanical uncertainty makes the motor system very flexible, but at the same time requires a very welldesigned controller that enables intelligent selection among the many possible alternatives. The theory of optimal control allows a principled approach to this problem - this assumes that the chosen mode of motion is based on the optimal task for the leg.
\end{abstract}

Keywords: optimal control; motor learning; prosthetic leg; reference tracking; active prosthetics; SmartLeg

\section{Motion properties of biological systems}

Biomechanical systems have a number of properties that differentiate them from the synthetic systems most commonly studied in control theory. It is very important for us to understand these characteristics in order to study the control of natural movements and to design control architectures to accomplish complex behaviours.

\subsection{High dimensionality}

One of the most significant features of biomechanical systems is that the state space has unusually high dimensionality. For example, consider a model with 2 joints and 6 muscles, which is often analysed in motor control, the condition space includes 2 joint angles and 2 joint speeds because it is a second order system. A realistic description of the condition should also include 6 muscle activations, as the muscles act as low pass filters of neural activity, with an insignificant time constant. For example, a similar approach for a complete model of the hand (including the palm) yields 20 dynamic states, and 50 muscle states. Such a state space cannot be discretized, which excludes all methods that rely on discretization. 


\subsection{Uncertainty and Noise}

Noise is a very important concept in modelling the biological processing of sensorimotor information. As early as 1954, Fitts [1] showed that fast-moving motions were driven by stochastic noise in neuromotor ducts: motor error variability increases with the magnitude of the movement. However, this is merely an interpretation of the effect of noise in terms of mathematical information theory, without a detailed quantitative contribution of the mechanisms responsible for random motion variability.

The considerable variability of biological movements indicates that the sensory system acts in the presence of major (mostly) internal disorders. Summation exists with both sensory information and motor commands. For example, noise in the sensory input will result in uncertainty in the position, and noise in the motor command describes the uncertainty of the actual force produced by the muscle [2], which will lead to inaccurate movement and variability.

The noise in the motor command is signal dependent - with the standard deviation increasing linearly with the magnitude of the motor command signal (control signal). This is a very important assumption, which is consistent with the observation recorded in Fitt's empirical law and supported by the empirical result from [2] and [3]. The optimal control of such systems should obviously take this phenomenon into consideration, since the appropriately selected control signal can actually reduce the noise. This model is widely used in the study of biological movements [4], [5], [6], [7], [8] and [9].

Our prosthesis is designed in a way to mimic leg movements in the sagittal plane. Its key features are knee and ankle joints which are movable in sagittal plane and are externally powered. Movements of knee and ankle enable obtaining required kinematics of the prosthesis, and powering system enables its dynamic features. Powered knee enables overcoming forces that occur during the swing phase. Powered ankle joint enables dorsal and plantar flexion movement of the entire foot, which provides better stabilization of the knee and the entire prosthesis and also provides power needed in push-off phase. In this paper we focus on the optimal control design of the active prosthetic leg in order to move in a biologically inspired way.

\section{Optimal performance criteria for movement planning}

The principle of optimality forms the basis of many physical sciences. In the field of motor control, the principles of optimality not only provide accurate descriptions of the observed phenomena but are also a priori justified. This is because the sensorimotor system is the product of an optimization process (i.e. evolution, development, learning, adaptation) that continuously improves behavioural performance. Thus, optimality provides an elegant framework for analysing system behaviour as well as determining the control laws that generate observed behaviour. When dealing with this optimal control problem, a special cost function must be selected to evaluate the efficiency of the system under control, and the goal is to reduce the value of this cost function.

Based on the observation that the trajectory represents a smooth function in the Cartesian coordinate space, Hogan [10] proposed a model of minimal movement in which the cost function depends on the square of the first derivation of Cartesian hand acceleration. That kind of cost functions depends on the kinematics of motion, and variables of interest include positions (e.g., joint angles or Cartesian hand coordinates), angular velocities, accelerations, and higher derivatives.

Let $x(t), y(t)$ denote the Cartesian coordinates of the hand position at time $t$, the minimum motion model is based on the following cost function:

$$
J=\frac{1}{2} \int_{0}^{T}\left(\left(\frac{d^{3} x}{d t^{3}}\right)^{2}+\left(\frac{d^{3} y}{d t^{3}}\right)^{2}\right) d t
$$

where $T$ is the duration of the motion. Assuming that the initial conditions are zero speed and acceleration, the optimal path takes the following form:

$$
\begin{aligned}
& x(t)=x_{0}+\left(x_{T}-x_{0}\right)\left(10\left(\frac{t}{T}\right)^{3}-15\left(\frac{t}{T}\right)^{4}+6\left(\frac{t}{T}\right)^{5}\right) \\
& y(t)=y_{0}+\left(y_{T}-y_{0}\right)\left(10\left(\frac{t}{T}\right)^{3}-15\left(\frac{t}{T}\right)^{4}+6\left(\frac{t}{T}\right)^{5}\right)
\end{aligned}
$$

where $\left(x_{0}, y_{0}\right)$ and $\left(x_{T}, y_{T}\right)$ are the start and end positions at time $t=0$ and $t=T$ respectively. From (2), it can be seen that the trajectory predicted by this criterion is a straight line with a parabolic shape of the velocity profile, which is consistent with empirical data for fast motion without limitation on accuracy. Since $x(t)$ and $y(t)$ depend only on the starting and 
ending positions of the arm and the movement time $T$, the optimal trajectory is determined only by the kinematics of the motion and is independent of the dynamics of the musculoskeletal system that generates movement.

By studying movements with a large range and observing a significant curvature of the trajectory of motion (which cannot be explained by the minimal motion model), Uno et al. [11] proposed an alternative model - a minimum momentchange model - in which trajectories were selected that optimize a function that sanctions the rate of momentum change.

This kind of cost function depends on the dynamics of the arm, and the variables of interest are the momentum change, the muscle tension, and the muscle command.

Let $\frac{d \tau_{i}}{d t}$ denote the rate of change of moment of the $i^{t h}$ joint, the model of minimum change of moment is based on the following cost function:

$$
\mathrm{J}=\frac{1}{2} \int_{0}^{\mathrm{T}} \sum_{\mathrm{i}=1}^{\mathrm{n}}\left(\frac{\mathrm{d} \tau_{\mathrm{i}}}{\mathrm{dt}}\right)^{2} \mathrm{dt}
$$

Because the minimum criterion for torque change depends on the dynamics of the leg, it can reproduce a gradually curved trajectory that cannot be explained on the basis of the minimum pitch model.

Although both models predict many aspects of trajectories consistent with empirical data and inspire a wealth of experimental and theoretical work, they still have several characteristics that make them unable to act as satisfactory motion models. These models consider only smooth paths, but do not suggest any advantage for smoothness of motion. In addition, there was no principled explanation for why the central nervous system was evaluated to optimize change in momentum or movement; and how the central nervous system was able to evaluate such complex values and integrate them over the duration of the trajectory is unknown.

\section{Combined system human - prosthesis}

Here, a method for modelling different stages of movement with the combined system human-prosthesis is described. Fig. 1 shows a kinematic hybrid transfemoral model, where the position of the prosthesis is shown in full, and the body is in a dashed line. The prosthetic leg is attached to the hip of the body. Dorsal and plantar flexion are defined in a positive or negative direction. First, the prosthetic leg will be modelled, followed by a hybrid human-prosthesis system.

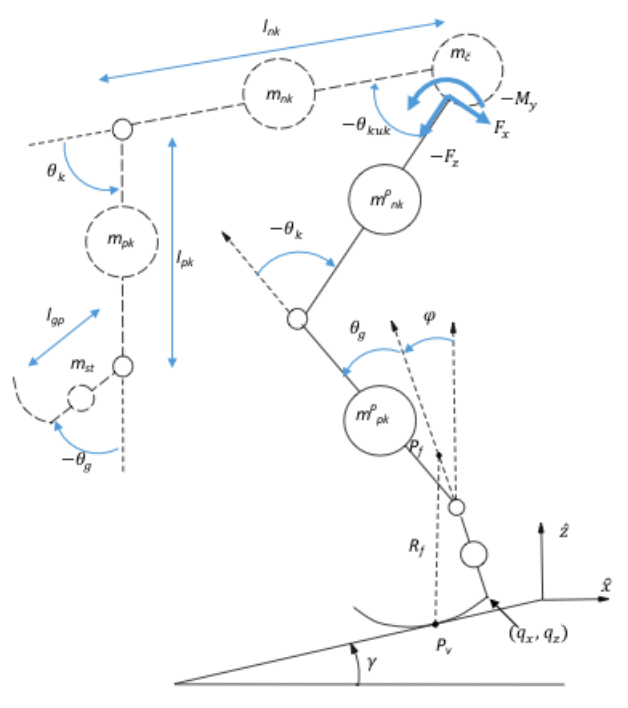

Fig. 1. Kinematic hybrid transfemoral model

\subsection{Dynamics}

The prosthetic leg is modelled as a kinematic chain in relation to the inertial reference coordinate system attached to the ground (Fig. 1). A movable coordinate system on the prosthetic heel is defined, treating its position coordinates $\left(q_{x}\right.$, $q_{z}$ ) as a state variable, which will be later constrained in the contact model. The complete configuration of the leg in the configuration space $Q=\mathbb{R}^{2} \times \mathbb{T}^{3}$ is given by $q=\left(q_{x}, q_{z}, \varphi, \theta_{a}, \theta_{k}\right)^{T}$, where $\varphi$ is the orientation of the lower leg relative to the vertical, $\theta_{a}$ is the angle in the ankle, and $\theta_{k}$ is the angle in the knee joint. 
The state of the dynamic system is then given by the vector $x=\left(q^{T}, \dot{q}^{T}\right)^{T} \in T Q$, where $\dot{q} \in \mathbb{R}^{5}$ contains the angular velocity of the joints. The trajectory of the state develops according to the differential equation of the form:

$$
\mathcal{M}(q) \ddot{q}+\mathcal{N}(q, \dot{q})+A(q)^{T} \lambda=B u+J(q)^{T} F
$$

where $\mathcal{M} \in \mathbb{R}^{5 \times 5}$ is an inertia/mass matrix, $\mathcal{N} \in \mathbb{R}^{5}$ is a vector that groups Coriolis/centrifugal members and gravitational forces, $A \in \mathbb{R}^{c \times 5}$ is a matrix that models physical constraints between feet and ground, and $\lambda \in \mathbb{R}^{c}$ is a Lagrange multiplier used to calculate contact forces.

The external forces on the right side of (4) contain the relevant moments of the drive motor and the interaction force with the body. The activation of the ankle and knee joints from the rotation momentum input $\lambda \in \mathbb{R}^{c}$ is mapped to the coordinate system of the leg over $B=\left(0_{2 \times 3}, I_{2 \times 2}\right)^{T} \in \mathbb{R}^{5 \times 2}$.

The interaction force $F=\left(F_{x}, F_{y}, M_{y}\right)^{T} \in \mathbb{R}^{3}$ is the force between the prosthesis and the corresponding part of the human leg (Fig. 1) and consists of two linear forces and one moment at the sagittal level, which can be measured with three-axes strain sensors at the prosthetic contact area. The vector force $\mathrm{F}$ acts at the end of the lower extremity kinematic chain and is mapped to the joint momentum/force of the body via the Jacobian matrix $J(q) \in \mathbb{R}^{3 \times 5}$, which is modelled by the standard procedure.

\subsection{Support phase}

During the period of standing, the physical forces associated with the contact between the prosthetic foot and the surface are modelled. These contact forces prevent the foot from slipping through the ground, which makes at least two physical constraints on the dynamics described in (4). Foot geometry is modelled as a holonomic constraint vector as described in [12], [13] and [14]:

$$
a(q)=0
$$

where $a: Q \rightarrow \mathbb{R}^{c}$ for $\mathrm{c} \geq 2$. The curved foot geometry is implemented (Fig. 1). Similarly, it is possible to implement other foot geometries, such as flat foot or foot with the bending in the metatarsophalangeal joint.

The contact constraint $a(q)=0$, yields the method for calculating the constraint matrix $A=\nabla_{q} a$ and the Lagrange multiplier $\lambda=\hat{\lambda}+\tilde{\lambda} u+\bar{\lambda} F$ :

$$
\begin{aligned}
& \hat{\lambda}=\mathrm{W}\left(\dot{\mathrm{A}} \dot{\mathrm{q}}-\mathrm{AM}^{-1} \mathcal{N}\right) \\
& \tilde{\lambda}=\mathrm{WAM}^{-1} \mathrm{~B} \\
& \bar{\lambda}=\mathrm{WAM}^{-1} \mathrm{~J}^{\mathrm{T}} \mathrm{T}
\end{aligned}
$$

for $W=\left(A \mathcal{M}^{-1} A^{T}\right)^{-1}$. These members enter the dynamics equation (4) only during the support phase, the period of resting of the prosthetic leg on the surface.

\subsection{Swing phase}

During the swing phase, the prosthetic foot is not in contact with the surface, so contact constraints do not appear in the dynamics of the prosthesis, i.e. $\lambda=0$ in (4). Although the prosthesis is still modelled with respect to its heel point, the interaction force $\mathrm{F}$ in the dynamics' equation (4) takes into account the action of the prosthetic leg on the hip.

\subsection{Transfemoral model}

In this paper, the case of a person with a one-sided transfemoral amputation will be considered, that moves using an active above-knee prosthesis with actuated knee and ankle joint. The hybrid human-prosthesis model (Fig. 2) consists of seven segments (links) of the leg and the point mass on the hip with the function of representing the upper part of the human body. The prosthesis is shown in a full black line and the human is shown in interrupted grey line. The general coordinates in the model are indicated by $q_{i}$. The angle $q_{1}$ is not actuated, and the angles $q_{2}-q_{6}$ have ideal actuators. The segments of the hip and thighs were modelled with rigid links with mass and inertia.

The model parameters are based on the dimensions of above-knee prosthesis prototype and subjects with amputation (Fig. 3). The model consists of an upper femoral part in the form of a prosthetic base, the lower tibial part and the prosthetic foot, the human subsystem, which consists of a contralateral thigh, below-the-knee part and foot, and the rest of the thigh on the amputated side and the point mass on the hip. It is assumed that the prosthetic part of the thigh and the rest of the human thigh are rigidly attached, so that the forces of interaction between them are of the same magnitude and opposite direction. 
Instead of modelling all contact phases and degrees of foot freedom, the foot and ankle function were modelled continuously using a curved foot and ankle joint to capture the positive momentum of the ankle position. This foot model involves rolling point contact, around which there is no moment of rotation, so that the reaction forces of the surface only contain tangential and normal components. Moreover, due to the fact that the foot rolls without slipping, the absolute angle $q_{1}$ is not actuated.

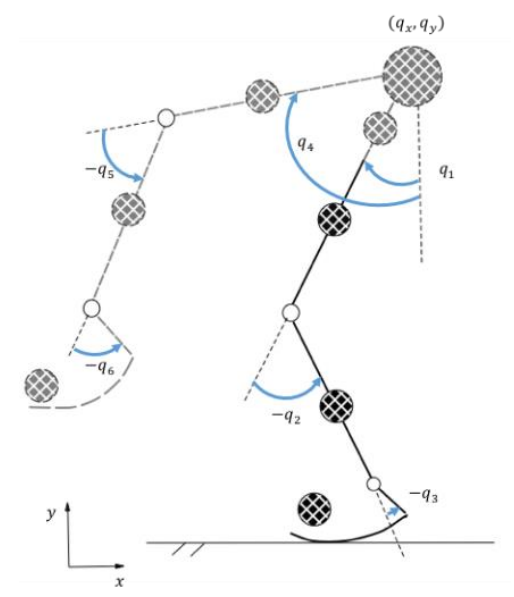

Fig. 2. Unilateral transfemoral hybrid model

In order to determine the position and the speed of the system, each subsystem has its own set of generalised coordinates. The configuration of each subsystem is described via the non-actuated angle $q_{1}$, the Cartesian coordinates of the hip $\left(q_{x}, q_{y}\right)$, and the relative angles of the actuated joints. Actuated joint angles for the whole system are $q_{2}-q_{6}$. Thus, the generalized coordinates are $q_{P}=\left[q_{1}, q_{2}, q_{3}, q_{x}, q_{y}\right]^{T}$ for prosthesis and $q_{H}=\left[q_{1}, q_{4}, q_{5}, q_{6}, q_{x}, q_{y}\right]^{T}$ for human. In addition, ideal actuators produce joint angular moments in $u_{P}=\left[u_{2}, u_{3}\right]^{T}$ for prosthesis and $u_{H}=$ $\left[u_{4}, u_{5}, u_{6}\right]^{T}$ for human.

For simulation, the step begins only after the transition from the contralateral position to the prosthesis' support phase, and this period continues after the prosthesis' support phase to the collision phase of the contralateral part. Two support phases can be modelled with continuous, differential equations of the second order, while two periods of impact can be modelled by algebraic mapping, which connects the state of the system immediately, with the moment after the impact. The equations of motion during the period of individual support phase can be written for each subsystem as follows:

$$
M_{i} \ddot{q}_{i}+C_{i} \dot{q}_{i}+N_{i}-E_{i}^{T} G_{i}=B_{i} u_{i}+J_{i}^{T} F
$$

where the index $i$ denotes the subsystem ( $P$ for prosthesis and $H$ for human), $q_{i}$ are the values of the coordinates of the subsystem, $M_{i}$ is the inertia matrix, $C_{i}$ is a matrix containing centripetal and Coriolis members, $N_{i}$ contains gravitational members, $E_{i}$ is the contact limit matrix, $G_{i}$ is a $2 \mathrm{D}$ vector force of the reaction of the substrate, $B_{i}$ transmits the angular momentum to the generalized coordinates, $J_{i}$ is the Jacobi matrix that connects the forces of interaction of the prosthetic socket with generalized coordinates, and $F$ is the $3 \mathrm{D}$ vector of the interaction force.

The solution for $\ddot{q}_{i}$ from the equation of motion (7) gives:

$$
\ddot{q}_{i}=M_{i}^{-1}\left(-C_{i} \dot{q}_{i}-N_{i}\right)+M_{i}^{-1} B_{i} u_{i}+M_{i}^{-1} J_{i}^{T} F+M_{i}^{-1} J_{i}^{T} G_{i}
$$

The equation (8) can also be written as a state space representation of the first order nonlinear system:

$$
\dot{x}_{i}=f_{i}\left(x_{i}\right)+g_{i}\left(x_{i}\right) u_{i}+p_{i}\left(x_{i}\right) F+r_{i}\left(x_{i}\right) G_{i}
$$

with

$$
\begin{aligned}
& x_{i}=\left[\begin{array}{c}
q_{i} \\
\dot{q}_{i}
\end{array}\right], f_{i}\left(x_{i}\right)=\left[\begin{array}{c}
\dot{q}_{i} \\
-M_{i}^{-1}\left(C_{i} \dot{q}_{i}+N_{i}\right)
\end{array}\right], g_{i}\left(x_{i}\right)=\left[\begin{array}{c}
0 \\
M_{i}^{-1} B_{i}
\end{array}\right], p_{i}\left(x_{i}\right)=\left[\begin{array}{c}
0 \\
-M_{i}^{-1} J_{i}^{T}
\end{array}\right] \\
& \text { and } r_{i}\left(x_{i}\right)=\left[\begin{array}{c}
0 \\
-M_{i}^{-1} E_{i}^{T}
\end{array}\right]
\end{aligned}
$$


The general coordinates $x_{i}$ are the states of a nonlinear system of the first order (9) with $f_{i}\left(x_{i}\right), g_{i}\left(x_{i}\right), p_{i}\left(x_{i}\right)$ and $r_{i}\left(x_{i}\right)$ as functions of a vector field that define a full dynamic system. Impacts can be modelled using the equations of the form:

$$
q_{i}^{+}=q_{i}^{-}, \dot{q}_{i}^{+}=A_{i} \dot{q}_{i}^{-}+\Lambda_{i} \mathcal{F}
$$

where $\mathcal{F}$ is the impulse of the interaction on the prosthetic socket that depends on the pre-impact state of both subsystems, and $A_{i}$ and $\Lambda_{i}$ are known matrices. Indices '-' and '+' refer to torque before and after the impact, respectively.

\section{Results and discussion}

The measurements refer to gait or walking phase of the human movement. It is performed by the human subject on one side and by the robotic leg on the other side using the healthy leg reference angles that are used as a setup for the optimal knee and ankle trajectories.

$P 3$ represents the prototype of the robotic above-knee prosthesis with separate hydraulic actuation in the knee and ankle joints. $I 1$ represents a healthy subject without amputation.

Results for the ankle joint are presented in the Fig. 3 and Table 1 and the results for the knee joint are presented in the Fig. 4 and Table 2.

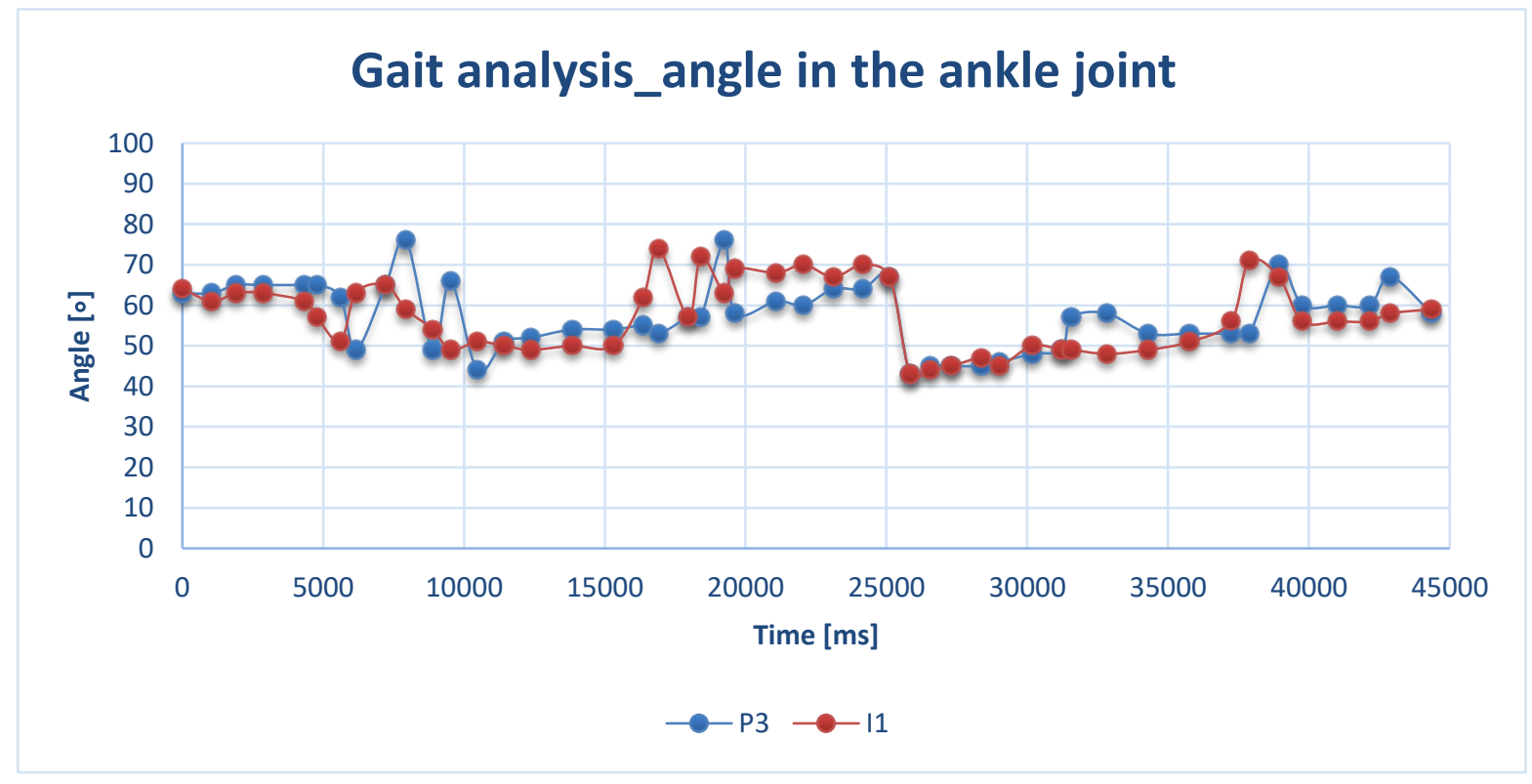

Fig. 3. Gait anlysis for the ankle joint

\begin{tabular}{|c|c|c|c|}
\hline \multirow{2}{*}{ Gait analysis } & $\mathbf{I 1}$ & $\mathbf{P 3}$ & \multirow{2}{*}{$\Delta$} \\
\cline { 2 - 3 } & $\boldsymbol{\varphi}_{\mathbf{3}}\left[^{\circ}\right]$ & $\boldsymbol{\varphi}_{\mathbf{3}}\left[^{\circ}\right]$ & \\
\hline $\begin{array}{c}\text { Average angle value } \\
(\boldsymbol{\mu})\end{array}$ & 57,404 & 57,510 & $0,18 \%$ \\
\hline Standard deviation $(\boldsymbol{\sigma})$ & 8,596 & 8,188 & $5 \%$ \\
\hline
\end{tabular}

Table 1. Gait analysis for ankle joint 


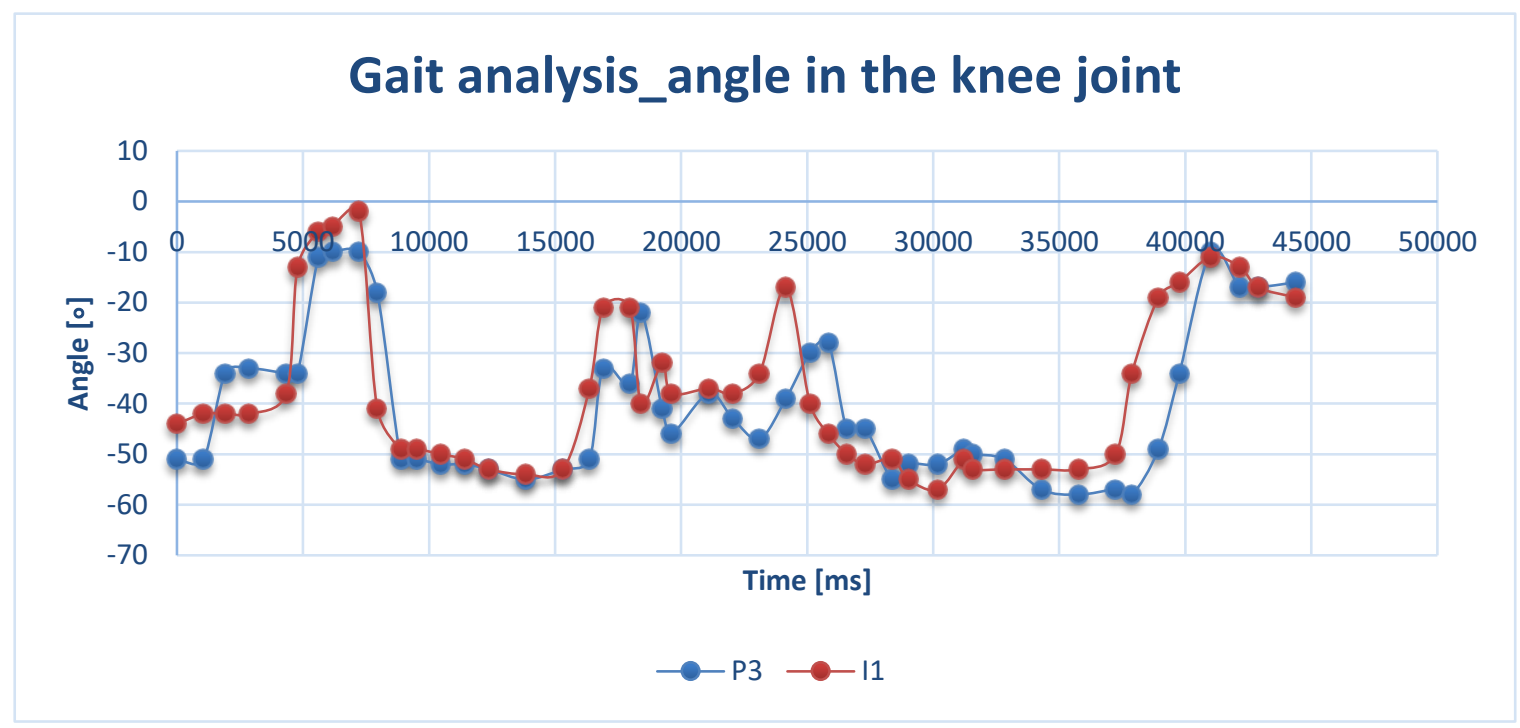

Fig. 4 Gait analysis for the knee joint

\begin{tabular}{|c|c|c|c|}
\hline \multirow{2}{*}{ Gait analysis } & I1 & $\mathbf{P 3}$ & \multirow{2}{*}{$\Delta$} \\
\hline & $\varphi_{2}\left[{ }^{\circ}\right]$ & $\varphi_{2}\left[^{\circ}\right]$ & \\
\hline $\begin{array}{c}\text { Average angle value } \\
(\mu)\end{array}$ & $-37,064$ & $-39,979$ & $7 \%$ \\
\hline Standard deviation $(\sigma)$ & 16,071 & 14,900 & $7 \%$ \\
\hline
\end{tabular}

Table 2. Gait analysis for knee joint

After the measurements were made, it was observed that small deviations occurred at maximum and the minimum values of the leg angles at the analysed leg movements. Deviations are not only present when comparing measurements of subjects without amputation and the autonomous movement of the prototype of the ankle prosthesis, but also in the analysis of change angles in different healthy subjects (subjects without amputation).

This occurs primarily because of the different leg shape among the subjects without amputation as well as subjects with amputation who used a prototype of the hip prosthesis. The other reason is the placement of sensors on each individual subject. Setting the sensor to the ankle prosthesis is different in that it can maximally avoid displacement via sensor fixation to the structure. This is not the case for subjects without amputation as the sensors are usually placed either on clothes or directly on the skin. In both cases the fixation is not rigid.

\section{Conclusion}

The latest suggested solutions for active prostheses of the lower limbs can significantly improve mobility and quality of life for millions of people with amputation of the lower extremities, but control challenges are currently limiting both clinical and commercial application and, therefore, the sustainability of these devices.

With the addition of sensors and motors, powered prosthetic legs must continuously make (optimal) control decisions during the gait cycle, thereby increasing the complexity of these devices. This complexity is usually solved by discretizing the gait cycle in several phases and time periods, each of which has its own separate control model. In the prosthesis with a greater number of joints, it is desired to control each joint independently. In doing so, each control model can follow the predefined trajectory of joint angles, speeds or torques.

In this paper, we present the real time optimal control strategy of newly developed above-knee prosthetic SmartLeg prototype with powered knee and ankle joints. Specialized control unit is developed in order to achieve required kinematics and dynamics to enable it to perform locomotion activities in a more natural manner. Future research will focus on optimization of the gait cycle and developing an optimal control for other locomotion tasks as climbing the stairs. 


\section{References}

[1] Fitts, P. M. (1954). The information capacity of the human motor system in controlling the amplitude of movement. Journal of Experimental Psychology, Vol.47, No.6, pp. 381-391

[2] Meyer, D. E.; Abrams, R. A.; Kornblum, S.; Wright, C. E. \& Smith, J. E. K. (1988). Optimality in human motor performance: Ideal control of rapid aimed movements. Psychological Review, 95, 340-370

[3] Schmidt, R. A.; Zelaznik, H.; Hawkin, B.; Frank, J. S. \& Quinn, J. T. (1979). Motor-output variability: a theory for the accuracy of rapid motor tasks. Psychological Review 86, 415-451

[4] Harris, C. M. \& Wolpert, D. M. (1998). Signal-dependent noise determines motor planning. Nature, Vol. 394

[5] Todorov, E. \& Jordan, M. (2002). Optimal feedback control as a theory of motor coordination. Nature Neuroscience, Vol.5, No.11, 1226-1235

[6] Todorov, E. \& Jordan, M. (2003). A minimal intervention principle for coordinated movement. Advances in Neural Information Processing Systems, 15: 27-34, Becker, Thrun, Obermayer (eds), MIT Press

[7] Todorov, E. \& Li, W. (2003). Optimal control methods suitable for biomechanical systems. Proceedings of the 25th Annual International Conference of the IEEE Engineering in Biology and Medicine Society

[8] Todorov, E. (2005). Stochastic optimal control and estimation methods adapted to the noise characteristics of the sensorimotor system. Neural Computation, 17(5), 2005.

[9] Jelačić, Z. Impedance control in the rehabilitation robotics, Book title: Advanced Technologies, Systems and Applications II, ISBN 978-3-319-71320-5, Publisher: Springer International Publishing AG, Copyright year: 2018.

[10] Dedić, R.; Huđec, M. \& Vučina, A. (2001). Simulation, Proceedings of the 12th DAAAM International Symposium "Intelligent Manufacturing \& Automation: Focus on precision engineering", 24-27 Octover, Jenna, Germany.

[11] Jelačić, Z. (2016). Motor Cognition and Decision Theory in Senzorimotor Control, ICAT Rome, 4th International Conference on Advanced Technology \& Sciences, 23-25 November 2016.

[12] Jelačić, Z. (2019). Wearable Sensor Control of Above-Knee Prosthetic Device. Acta Scientific Orthopaedics 2.7: 02-11. (Published: July, 2019)

[13] Jelačić Z.; Dedić R. (2020). Reference Tracking of the Robotic Above-Knee Prosthetic Leg with Actuated Knee and Ankle Joints Using Robust Control. In: Badnjevic A., Škrbić R., Gurbeta Pokvić L. (eds) CMBEBIH 2019. CMBEBIH 2019. IFMBE Proceedings, vol 73, pp 287-292 